\title{
Neural Network Controller for Minimizing Hub Shear Forces in Helicopter
}

\author{
S.N.Omkar and J.Nagabhushanam \\ Department of Aerospace Engineering \\ Indian Institute of Science \\ Bangalore, India 560012 \\ e-mail:omkar@aero.iisc.ernet.in
}

\begin{abstract}
This paper discusses the application of recurrent neural networks for identification and control of helicopter vibrations. A class of recurrent networks called Memory Neuron Networks are used for plant identification and control. These networks are obtained by adding trainable temporal elements to feed-forward networks. This makes the network output history sensitive and gives them the capability to identify and control systems whose order is unknown or systems with unknown delay. A representative analytical model with higher harmonic pitch angles for minimizing hub shear forces is used for simulation. The effectiveness of the controller in minimizing the force level at varying and constant forward speed are studied. The ability of the controller to cope with changes in system and environment parameters is also considered.
\end{abstract}

\section{KEY WORDS: neural networks, adaptive controllers, helicopters}

\section{INTRODUCTION}

Vibrations in helicopters are caused by a variety of sources, such as rotor systems, engine, transmission etc. Rotor blades which operate in complex aerodynamic environment form the chief source of these vibrations. The nearly periodic blade loads are transmitted to the fuselage via the blade-root forces. The transmitted forces result in fuselage vibrations, principally at a rate of ' $n$ ' per revolution, where ' $n$ ' is the number of blades. Vibratory loads lead to fatigue damage of structural components, human discomfort, difficulty in reading instruments and reduced effectiveness of weapon systems. These oscillatory loads are often sensitive to the design parameters of the rotor and fuselage system. Furthermore, these loads increase with forward speed and depend on flight conditions. With increasing demand for high speed and high maneuverability for both military and civilian applications, vibration control in helicopters has become a major issue.

Passive vibration control devices are commonly used in which vibration level is either isolated(isolators) or the vibration level is diffused (absorbers). The major drawback with the passive devices is their limited performance advantage for a narrow range of flight conditions.

Higher Harmonic Control (HHC) is one of the active control techniques in which the higher harmonic components of the pitch angle are deployed to reduce the rotor unsteady aerodynamic forces at the very source. This reduces the transmitted forces resulting in the minimization of vibration levels.

Considerable theoretical and experimental work has been done in active control vibration reduction techniques for helicopters $[5,4,6,12]$. In these vibration reduction systems control schemes known as multicyclic control [5] or higher harmonic control [6] have been applied to reduction of inherent vibratory responses of a helicopter. Typically the helicopter is represented by a linear quasi-static frequency domain model. The relationship between control inputs and outputs (which include vibrations, loads, and rotor performance parameters) are modeled by a transfer function matrix. Theoretical and experimental results show that the vibratory level of a helicopter can be significantly reduced by using controllers in which the transfer function matrix is updated by a Kalman filtering scheme. These vibration reduction systems are reviewed in detail in Ref [12].

Helicopters operate in a complex aerodynamic environment with requirements of high maneuverability, high speed and multi-role capabilities. Hence the successful operation of a flight-worthy vibration control system depends on its ability to cope with uncertainties that may arise within the system and the environment; its ability to accurately capture the nonlinearities and its processing speed to cater for computational complexities. Artificial Neural Networks (ANNs) are capable of meeting all these difficulties encountered in a complex control system [10]. It is known that they can approximate arbitrary nonlinear maps from one finite dimensional space to another. Methods for adjusting their parameters on the basis of input-output data to cope with uncertainty are also available. Finally, their parallel distributed architecture makes them attractive for coping with computational complexity. It would also be desirable that controller can autonomously improve upon its performance as experience is gathered [1]. This implies that the network should have memory to exploit past experience to improve future performance.

Several ANN architectures have been successfully applied for identification and adaptive control of nonlinear dynamical systems $[8,13,3,14]$. ANNs are finding wide range of applications in the field of helicopters. Recently, studies have been done $[7,2]$ on helicopter flight control in which a nominal inverting controller is designed based on a single flight simulation, with 
augmentation provided by an adaptive neural network. In this paper, a recurrent neural network called Memory Neuron Network (MNN) [3] is exploited to develop an adaptive controller to vary higher order harmonic pitch components so as to minimize rotor forces transmitted to the fuselage.

\section{MEMORY NEURCN NETWORK(MNN)}

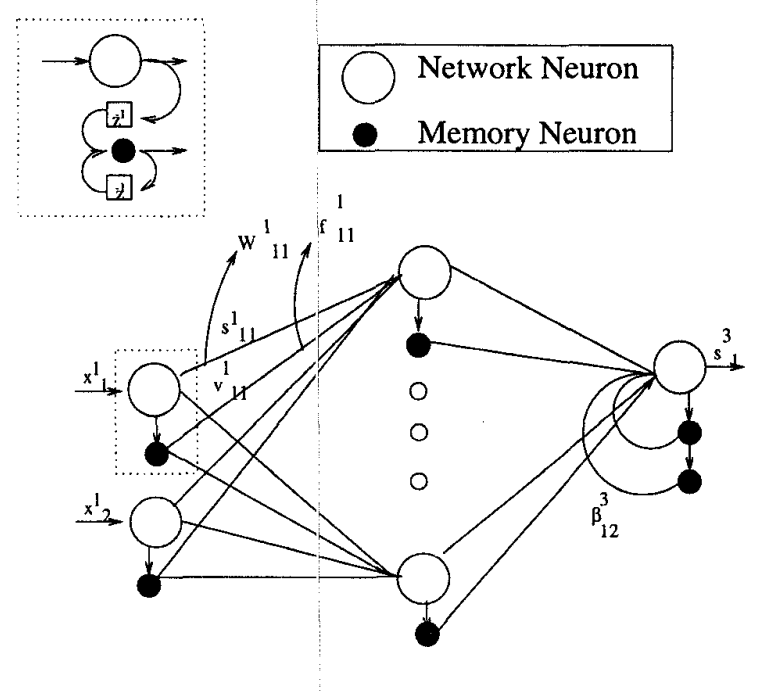

Figure 1. Architecture of a MNN

The architecture of a NINN is shown in Figure 1. The notations used are:

$L$ is the number of layers of the network with layer 1 as the input layer and layer $L$ as the ouiput layer.

$x_{j}^{l}(k)$ is the net input to the $j^{\text {th }}$ network neuron of layer $l$ at time $k$.

$s_{j}^{l}(k)$ is the output of the $j^{t h}$ network neuron of layer $l$ at time $k$.

$v_{j}^{l}(k)$ is the output of the memory neuron of the $j^{\text {th }}$ network neuron in layer $l$ at time $k, 1 \leq l<L$.

$w_{i j}^{l}(k)$ is the connectin $;$ weight from the $i^{t h}$ network neuron of layer $l$ to the $j^{\text {th }}$ network neuron of layer $l+1$ at time $k$.

$f_{i j}^{l}(k)$ is the connecting weight from the memory neuron of the $i^{\text {th }}$ network neuron of layer $l$ to the $j^{\text {th }}$ network neuron of layer $l+1$ at time $k$.

$\alpha_{j}^{l}(k)$ is the connecting weight from the $j^{t h}$ network neuron to its corresponding memory neuron at time $k$.

$\alpha_{i j}^{L}(k)$ is the connecting weight from the $(j-1)^{t h}$ memory neuron to the $j^{\text {th }}$ memory neuron of the $i^{\text {th }}$ network neuron in the output layer at time $k$.

$v_{i j}^{L}(k)$ is the output of the $j^{\text {th }}$ memory neuron of the $i^{\text {th }}$ network neuron in the output layer at time $k$.

$\beta_{i j}^{L}(k)$ is the connecting, weight from the $j^{\text {th }}$ memory neuron of the $i^{\text {th }}$ network neuron to the $i^{\text {th }}$ network neuron in the output layer at time $k$.

The structure is the same as feed forward ANN except for the memory neurons (shown by small filled circle) attached to each unit in the network (shown by large open circles). As can be seen from the figure, at each level of the network except the output level, each of the network neurons has exactly one memory neuron connected to it. The memory neuron takes its input from corresponding network neuron and it also has a self feedback as shown in the inset of figure 1. This leads to accumulation of past data of the network neuron in the memory neuron. The main features of these networks are: they have trainable internal memory to directly model dynamical systems; can model systems of unknown order; the architecture and the learning algorithms are closely related to the well proven feed forward networks. The details of the learning algorithm, identification and control architectures are discussed in reference [3].

\section{ANALYTICAL MODEL}

For the purpose of simulation a simple analytical model is considered. The model consists a three bladed rotor having rigid flap-lag motions and a fuselage with rigid pitch and roll motions with higher harmonic control. The following parameters are used for the model: solidity $\sigma=0.05$, Lock number $\gamma=5$, rotating-flap natural frequency $\omega_{\beta}=1.15$, rotating-lag natural frequency $\omega_{\zeta}=0.7$, vertical distance between rotor hub and fuselage C.G., $\bar{h}=0.25$, flat-plate area $\bar{f}=0.01$, profile drag coefficient $C_{d 0}=0.01$, thrust coefficient $C_{T}=0.01$, nondimensional inertias of Roll and Pitch 3.24 and 12.96 respectively. The forward speed of the model is nondimensionalised with respect to the tip speed of the rotor and is called advance ratio $(\mu) . \mu$ varies from 0 to 0.4 . The control of the analytical model is provided by the blade pitch angle $\theta$ which consists of two contributions; those needed to trim the helicopter and those needed for the higher harmonic pitch inputs used for minimizing hub shear forces. This can be expressed as:

$$
\theta=\theta_{o}+\theta_{c} \cos \psi+\theta_{s} \sin \psi+\sum_{j=2}^{3}\left(\theta_{j c} \cos j \psi+\theta_{j s} \sin j \psi\right)
$$

The higher harmonic pitch vector used for control of vibrations can be written as:

$$
\theta_{H H C}=\left\{\theta_{2 c} \theta_{2 s} \theta_{3 c} \theta_{3 s}\right\}
$$

In this study the cosine and sine components of 3-per-rev hub forces constitute the vector $\mathcal{Z}$.

$$
\mathcal{Z}=\left\{F_{x 3 c} F_{x 3 s} F_{y 3 c} F_{y 3 s} F_{z 3 c} F_{z 3 s}\right\}
$$

It may be noted that $\mathcal{Z}$ is a function of not only $\theta_{H H C}$ but also $\mu, C_{T}$ and other rotor-fuselage parameters. An objective function to be minimized is chosen as

$$
J=\sqrt{\frac{1}{6}\left\{F_{x 3 c}^{2}+F_{x 3 c}^{2}+F_{y 3 c}^{2}+F_{y 3 c}^{2}+F_{z 3 c}^{2}+F_{z 3 c}^{2}\right\}}
$$


The uncontrolled $\mathrm{J}$ and $\theta_{H H C}$ for minimizing $\mathrm{J}$ can be analytically evaluated $[9,11]$ for comparing with NN Controller.

\section{SIMULATION}

A series-parallel model and model reference adaptive control architecture are used [3]. Since the force level ideally needed is zero, the output of the reference model is set to zero. The identification model has $\theta_{H H C}$ as input and $\mathcal{Z}$ as the output. It has two hidden layers with 60 nodes and 40 nodes respectively and each output node has 3 memory neurons. The controller model has $\mathcal{Z}$ as its input and $\theta_{H H C}$ as output. It has 40 nodes and 20 nodes respectively in first and second hidden layers with 3 output memory neurons. The learning rate used for network neurons is 0.3 and for memory neurons is 0.2 . Two types of activation functions are used.

$$
\begin{aligned}
& g_{1}(x)=\frac{1}{1+\exp (-x)} \\
& g_{2}(x)=\frac{1-\exp (-x)}{1+\exp (-x)}
\end{aligned}
$$

$g_{1}(x)$ is used for all hidden nodes and $g_{2}(x)$ is used for the output nodes. Some more parametric studies are underway to arrive at an optimum configuration.

For identification, data is generated using analytical model, by varying advance ratio from 0 to 0.4 over 100 cycles. At each cycle random values for $\theta_{H H C}$ are given and the corresponding force vector $\mathcal{Z}$ is calculated. This set of values consisting of only $\theta_{H H C}$ and $\mathcal{Z}$ are used to train the MNN identification model. About 300 epochs of offline training is found sufficient. For testing the identification model, speed is varied from 0 to 0.4 for 200 cycles setting $\theta_{H H C}$ to zero. The results of the identification are shown in figure 2 . The output of the identifier network is in excellent agreement with the analytically predicted results.

Controller network is trained by putting the analytical model on line. Advance ratio is varied from 0 to 0.4 in 500 cycles to train the controller. The effectiveness of the controller in minimizing the objective function $(\mathrm{J})$ is tested in the following ways.

1. The controller is switched on and the forward speed is varied from 0 to 0.4 over 200 cycles. Figure 3 shows the minimized objective function. In comparison with figure 2 (uncontrolled J), it can be seen that the controller is quite effective in reducing the objective function.

2. The analytical model is flown at a constant advance ratio of 0.4 . Then the controller is switched on. Figure 4 shows the controlled $\mathrm{J}$ over 200 cycles. It can be seen that the controller is very effective right from the beginning and good minimization is reached in first few cycles.

3. The analytical model is flown at a advance ratio of 0.3 with the controller on. At 75 th cycle the advance ratio is suddenly increased to 0.4 and maintained till 125 th cycle after which advance ratio is reduced to 0.3 . Figure 5 shows the effectiveness of controller in adapting to changes in the operating conditions.

4. One of the important model parameter is gross weight $\left(C_{T}\right)$ to be lifted. This has a bearing on both $\theta_{H H C}$ and $\mathcal{Z}$. It may be noted that the network is trained for $C_{T}=$ 0.01 . The analytical model is flown at $\mu=0.4$. At 100 th cycle $C_{T}$ is reduced to 0.006 . It can be seen from Figure 6 that the controller further reduces $J$ after 100 th cycle as can be expected.

5. The $\mathcal{Z}$ vector consists of 6 components of which the last two are quite dominant. To test the controller's ability to cope up with failure of sensor, one of the dominant component of $\mathcal{Z}$ is set to zero. The analytical model is flown at $\mu=0.3$ and the 5 th component of $\mathcal{Z}$ is set to zero at 100 th cycle. Figure 7 shows that the controller successfully copes up with the failure of one of the sensors.

\section{CONCLUSIONS}

In this paper a recurrent neural network namely Memory Neuron Network is successfully used for identification and control of forces transferred from helicopter rotor to fuselage. The simulations show that the controller is efficient in reducing the force level and that it can cope with changes in system and environment parameters. The simulations also show that the controller is capable of handling failure of some measuring equipments. Some more theoretical and experimental investigations are needed to make it a flight-worthy controller. However, the simulations provide a pointer towards the potential of MNN as an intelligent controller.

\section{References}

[1] K.J. Astrom and Thomas J. McAvoy, Intelligent control-an overview and evaluation, VAN NOSTRAND REINHOLD, 1992.

[2] Jesse Leitner,Anthony Calise and J.V.R.Prasad, Analysis of adaptive neural networks for helicopter fight control, Journal of Guidance, Control, and Dynamics 20 (1997), no. 5, 972-979.

[3] P.S.Sastry, G.Santharam and K.P.Unnikrishnan, Memory neuron networks for identification and control of dynamical systems, IEEE Transaction on Neural Networks 5 (1994), no. 2 . 
[4] Chopra I. and McCloud III.J.L, Considerations of openloop, closed-loop and adaptive multicyclic control systems, American Helicopter Society 28 (1983), no. 1.

[5] McCloud III.J.L, The promise of multicyclic control for helicopter vibration reluction, Vertica 4 (1980), 29-41.

[6] Shaw J., Higher harmonic blade pitch control: a system for helicopter vibration reduction, Ph.D. thesis, MIT, Cambridge,MA, 1980.

[7] Kim, B.S and Calise, A.J, Nonlinear flight control using neural networks, Journal of Guidance, Control, and Dynamics 20 (1997), nc. 1, 26-33.

[8] K.S.Narendra and K.Parthasarathy, Identification and control of dynamical systems using neural networks, IEEE Transaction on Neural Networks 3 (1990), no. 2, 4-27.

[9] I.Papavassiliou, P.P.Friedmann and C.Venkatesan, Coupled rotor-fuselage vibration reduction using open-loop blade pitch control, Mathl. Comput. Modelling 18 (1993), no. $3 / 4,131-156$.
[10] Kumpati S.Narendra and Snehasis Mukhopadhyay, Intelligent control using neural networks, Intelligent Control Systems- Theory and Applications (Madan M.Gupta and Naresh K.Sinha, eds.), IEEE Press, 1996.

[11] S.N.Omkar and J.Nagabhushanam, Simultaneous computation of trim and higher harmonic controls of helicopter, National Seminar on Aerospace Structures -NASAS (Bangalore,India), 1997.

[12] Johnson W., Self-tuning regulators for multicyclic control of helicopter vibration, 1982, NASA TP-1996.

[13] Y.Ichikawa and T.Ssawa, Neural networks application for direct feedback controllers, IEEE Transaction on Neural Networks 3 (1991), no. 2, 252-262.

[14] Hunt, K.J., Sbarbaro,D., Zbikowski,R. and Gawthrop, P.J., Neural networks for control systems: A survey, Automatica 28 (1992), no. 6, 1083-1112. 


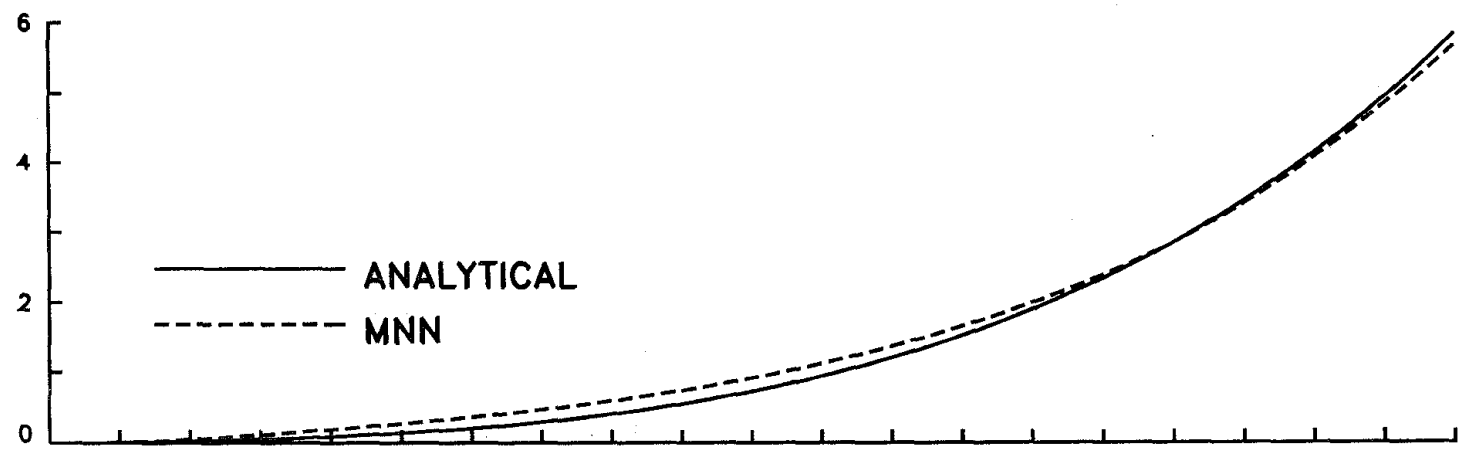

Fig.2: Identification of uncontrolled $J$ for $\mu$ varying from 0 to 0.4

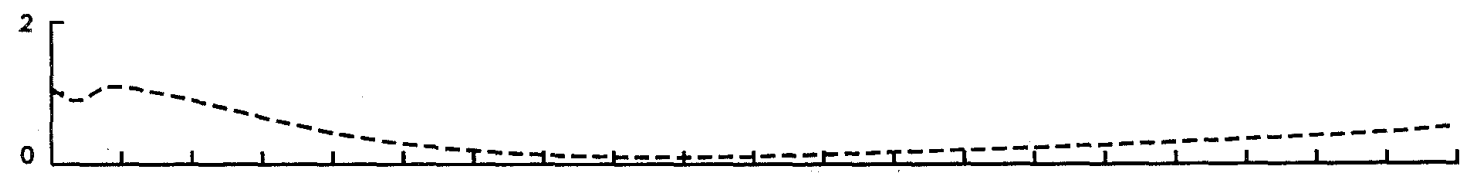

Fig.3: Controlled $\mathrm{J}$ for $\mu$ varying from 0 to 0.4 in 200 cycles

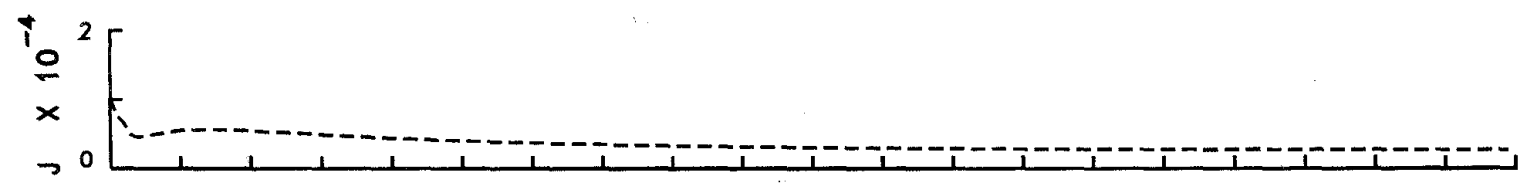

Fig.4: Controlled $J$ for constant $\mu$ in 200 oycles

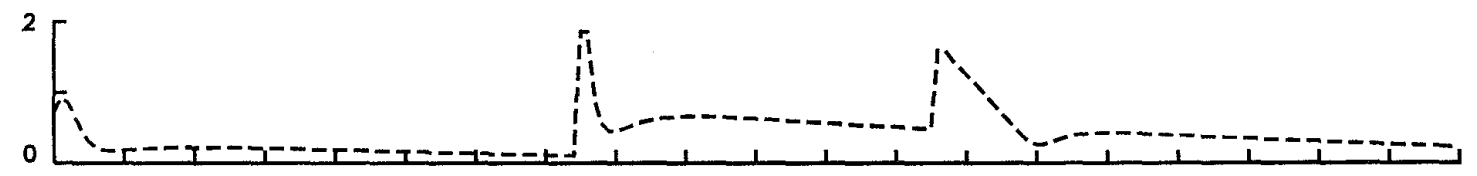

Fig.5: Controlled $J$ for $\mu$ changing from .3 to .4 at 75 th cycle and from .4 to .3 at 125 th cyle

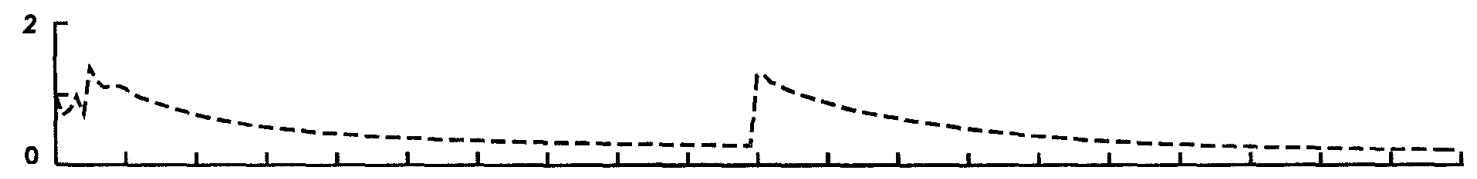

Fig.6: Controlled $J$ for $\mu=0.4$ and $C_{T}$ changing from 0.01 to 0.006

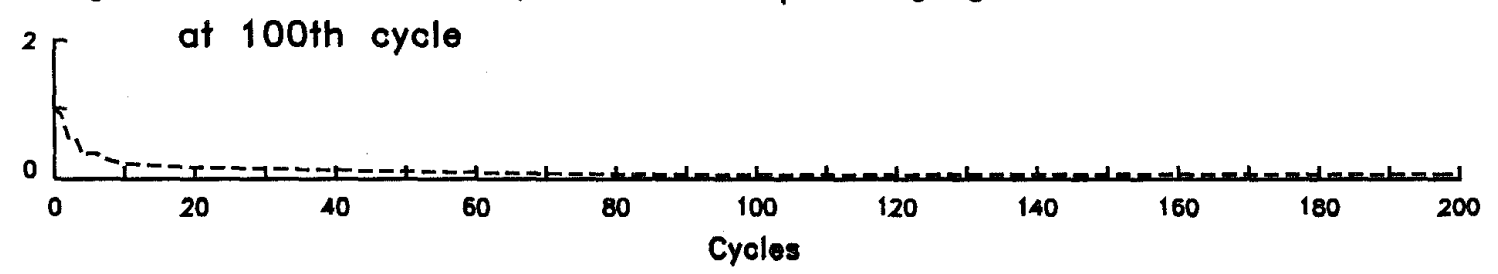

Fig.7: Controlled $J$ for $\mu=0.3$ with one of the sensors failing at 100th cycle 